\title{
Early assessment of infarct size by feature tracking cardiac MRI in patients with myocardial infarction
}

\author{
Sebastian Buss ${ }^{1 *}$, Birgit Krautz ${ }^{1}$, Nina P Hofmann ${ }^{1}$, Kristin Breuninger ${ }^{1}$, Yannick Sander ${ }^{1}$, Rebekka Kammerer ${ }^{1}$, \\ Philipp Matheis ${ }^{1}$, Lukas Rust ${ }^{1}$, Christian Galuschky², Philip Raake1, Sven T Pleger ${ }^{1}$, Grigorios Korosoglou'
}

From 16th Annual SCMR Scientific Sessions

San Francisco, CA, USA. 31 January - 3 February 2013

\section{Background}

The degree and quantification of contractile dysfunction and myocardial scar in patients after acute myocardial infarction (AMI) has important prognostic implications. Myocardial deformation parameters like strain and strain rate have been shown to be more sensitive markers for contractile dysfunction than standard 2-D parameters.

We sought to investigate whether strain and strain rate imaging, assessed by a novel non-invasive post-processing feature tracking algorithm (FTI) on pre-acquired regular CMR SSFP images, would allow quantification of regional left ventricular (LV) function and its relation to degrees of infarct trans-murality in patients after AMI.

\section{Methods}

Cardiac magnetic resonance (CMR) imaging was performed on a $1.5 \mathrm{~T}$ whole body MRI scanner (Philips Achieva) in 44 patients (mean age: $56 \pm 12$ ) $3 \pm 1$ days after successfully reperfused first ST segment elevation AMI. Peak systolic circumferential strain and strain rate were measured on standard CMR SSFP cine sequences and afterwards related to the trans-mural extent of myocardial infarction determined by contrast enhanced CMR (CE-CMR) 10 minutes after i.v. injection of $0.2 \mathrm{mmol} / \mathrm{kg}$ Gd-DTPA (Magnevist). The feature tracking algorithm (2D CPA MR $\odot$, TomTec Imaging Systems GmbH) is a two dimensional deformation analysis of the myocardium that was originally designed for echocardiographic image analysis, which has now been transferred to CMR SSFP sequences without the need for additional scans.

${ }^{1}$ University of Heidelberg, Heidelberg, Germany

Full list of author information is available at the end of the article

\section{Results}

Cut-off peak systolic circumferential strain values of $-21 \%$ differentiated patients with global infarct sizes $\geq 20 \mathrm{~g}$ from patients with smaller infarct sizes providing sensitivities and specificities of $94 \%$ and $74 \%$. An infarcted myocardium with a segmental infarct size of $\geq 50 \%$ was differentiated from non-transmural infarcted segments with a cut-off peak systolic circumferential strain value of $>-14 \%$ with a sensitivity of $92 \%$ and a specificity of $86 \%$. Peak negative circumferential strain correlated closely with the quantitatively determined infarct size on a global $(r=0.78, p<0.001)$ and segmental level $(\mathrm{r}=0.76, \mathrm{p}<0.001)$.

\section{Conclusions}

FTI provides a rapid and objective quantification of global and regional myocardial function and allows the discrimination between different degrees of trans-murality in patients after AMI.

\section{Funding}

none

\section{Author details}

${ }^{1}$ University of Heidelberg, Heidelberg, Germany. ${ }^{2}$ TomTec Imaging Systems, Munich, Germany.

Published: 30 January 2013

doi:10.1186/1532-429X-15-S1-P196

Cite this article as: Buss et al.: Early assessment of infarct size by feature tracking cardiac MRI in patients with myocardial infarction. Journal of Cardiovascular Magnetic Resonance 2013 15(Suppl 1):P196. 